\title{
Spontaneous extrusion of porous amphiphilic triblock copolymeric microfibers under microfluidic conditions
}

\author{
Polymer Journal (2010) 42, 100-102; doi:10.1038/pj.2009.301
}

During the last decade, nano- and microfibers obtained from biodegradable polymers have received much attention in medical applications, such as tissue engineering and drug delivery systems. ${ }^{1,2}$ A variety of applications could be possible if the fibers contained porous structures; for example, in the case of drug/protein-loaded fibers, porous surface features are required for controlled release to maintain bioavailability or specific tissue regeneration. Furthermore, microporous fibers can provide more anchoring points for cell accommodation, maintain cell survival and facilitate the diffusion of nutrients in tissue regeneration.

The release of drugs from fibrous carriers can be designed to be rapid or delayed, depending on the polymer carrier used. Therefore, amphiphilic copolymers have received increasing attention in medical applications as they possess hydrophilic and hydrophobic segments, which facilitate either water-soluble or lipid-soluble drug release. Even though amphiphilic copolymers have a wide range of applications, to the best of our knowledge, there are no reports in the literature on fabricating microfibers made of amphiphilic triblock copolymers using microfluidic phenomena. For the first time, we have synthesized and used the amphiphilic triblock copolymer poly (p-dioxanone-cocaprolactone)-block-poly (ethylene oxide)block-poly (p-dioxanone-co-caprolactone) (PPDO-co-PCL-b-PEG-b-PPDO-co-PCL) for continuous porous microfiber fabrication using a microfluidic device. This copolymeric carrier has a hydrophilic PEG segment and a hydrophobic PPDO/PCL segment, and researchers have already suggested that it has good drug encapsulation ability. ${ }^{4}$ The microfluidic device used has a wide range of advantages: microfibers can be produced continuously in a simple, cost-effective manner, and the process is carried out under ambient conditions without the use of high temperatures and pressures. Therefore, sensitive biomaterials, such as proteins, DNA or cells, can be easily immobilized or entrapped in the microfibers for use in sensing, drug delivery and tissue-engineering applications. The scaffold was characterized in terms of its morphological porous structure, capability for drug release and hydrophilicity to determine cell adhesion ability.

\section{EXPERIMENTAL PROCEDURE}

PPDO-co-PCL-b-PEG-b-PPDO-co-PCL amphiphilic triblock copolymer was synthesized according to the procedure developed by Bahadur et al. ${ }^{3}$ The composition, average

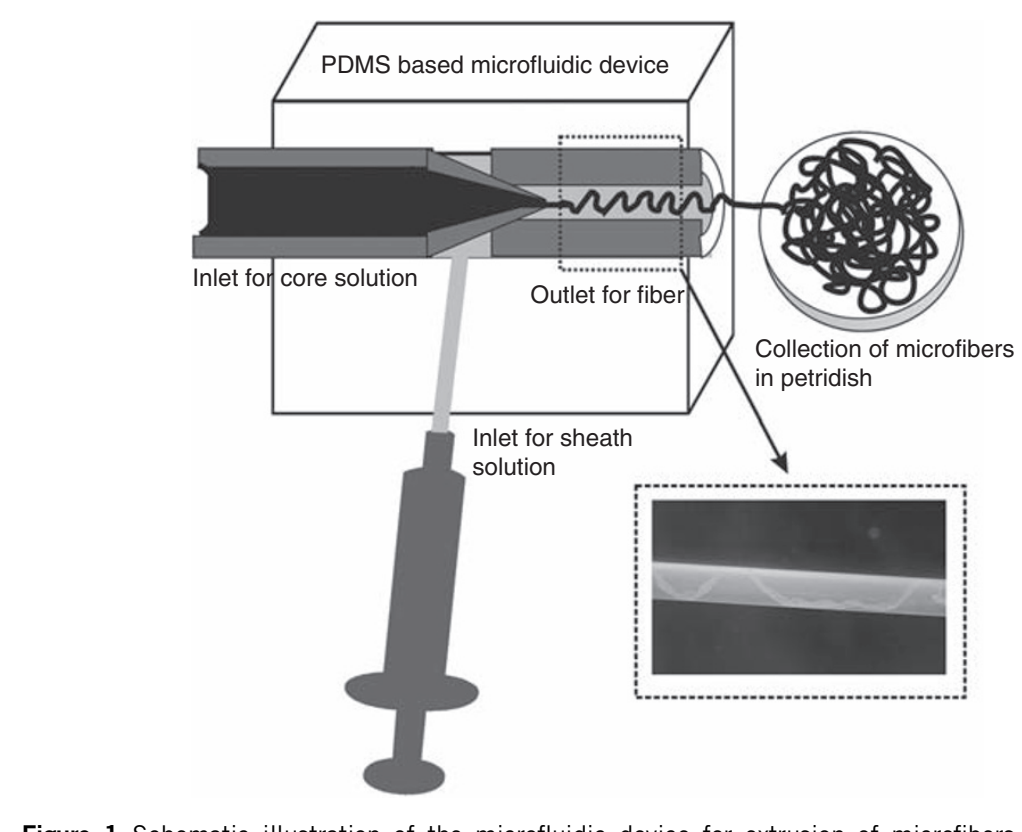

Figure 1 Schematic illustration of the microfluidic device for extrusion of microfibers (inset: optical micrograph of fiber from capillary outlet). molecular weights $\left(M_{\mathrm{w}}\right)$, polydispersity index $\left(M_{\mathrm{w}} / M_{\mathrm{n}}\right)$, melting temperature (Tm) and glass transition temperature $(\mathrm{Tg})$ of the copolymer are 25:55:20 (PEG:PCL:PPDO), $45.9 \times 10^{3} \mathrm{~g} \mathrm{~mol}^{-1}, 1.5,48.58^{\circ} \mathrm{C}$ and $-58.16^{\circ} \mathrm{C}$, respectively. A microfluidic-based device was constructed using the established methods of pipette pulling and polydimethylsiloxane molding, ${ }^{4}$ with slight modifications as schematically explained in Figure 1. In our microfluidic system, the inner diameter of the core inlet micropipette (50 and $25 \mu \mathrm{m}$ in diameter at the outer end and at the pulled end, respectively) was two times larger than that of the outlet micropipette $(25 \mu \mathrm{m}$ at both ends). The core solution (10\% PPDO-coPCL-b-PEG-b-PPDO-co-PCL copolymer in $\mathrm{CH}_{2} \mathrm{Cl}_{2}$ or in dimethylsulfoxide (DMSO)) 


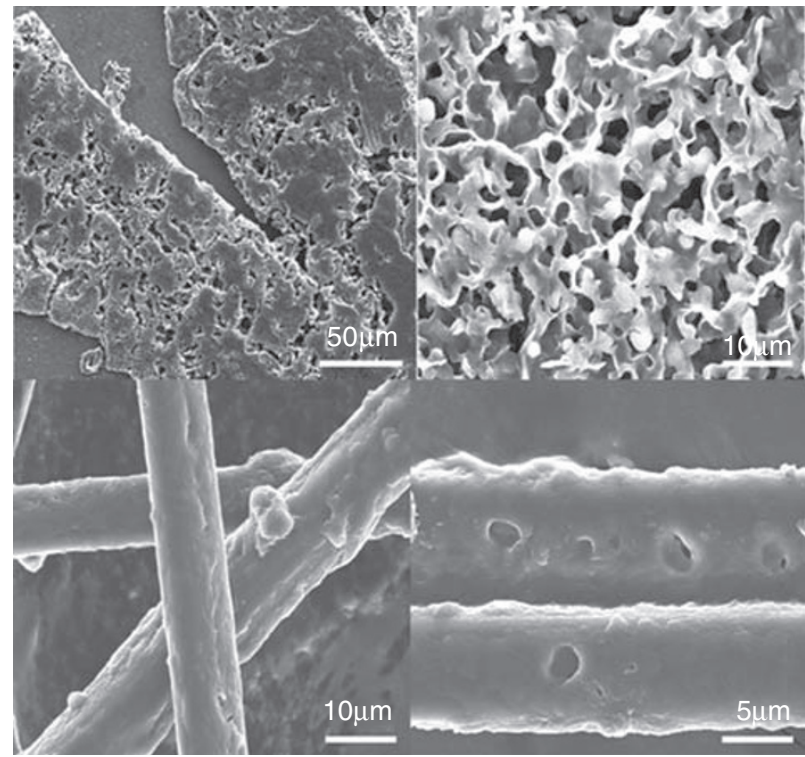

Figure 2 Field electron scanning electron microscopy images of porous microfibers extruded using $\mathrm{CH}_{2} \mathrm{Cl}_{2}(20 \mu \mathrm{m}$ in diameter (a)) and dimethylsulfoxide $(5-10 \mu \mathrm{m}$ in diameter (b)) as solvents.

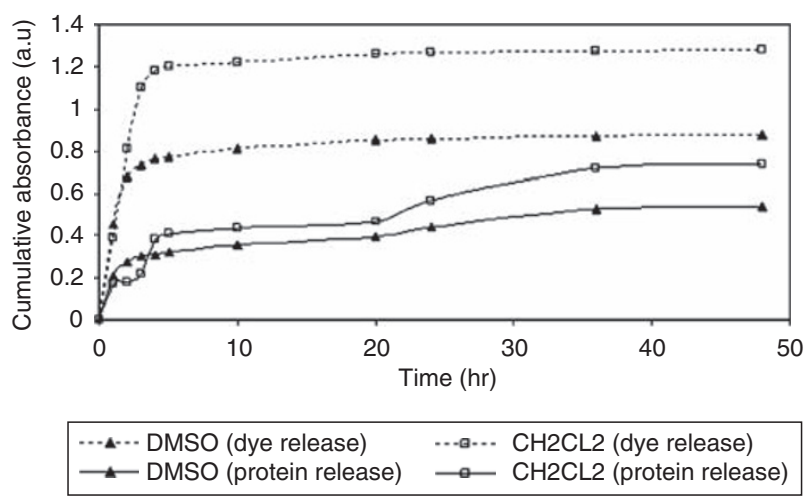

Figure 3 Cumulative release profiles of drug and dye from microfibers.

Table 1 Surface roughness and hydrophilicity of the fibers

\begin{tabular}{lcc}
\hline Properties & Microfiber & Microfiber \\
\hline Roughness & $1.801 \mu \mathrm{m}$ & $1.099 \mu \mathrm{m}$ \\
Contact angle (degree) & & \\
$\quad$ Sterilized $\mathrm{H}_{2} \mathrm{O}$ & 28 & 30 \\
Cell suspension (mouse fibroblast cells & 32 & 35 \\
with $2 \times 10^{5}$ cells ml-1) & & \\
\hline
\end{tabular}

${ }^{\mathrm{a}} \mathrm{CH}_{2} \mathrm{Cl}_{2}$ solvent system.

bimethylsulfoxide solvent system. and sheath solution (DI water) were injected through the micropipette inlet with a 12gauge needle inlet with infusion pumps, and their flow rates were maintained at $40 \mu \mathrm{min}^{-1}$ and $50 \mathrm{ml} \mathrm{h}^{-1}$, respectively. The extruded porous fibers were collected in a precleaned Petri dish. microfluidic device and surface chemistry profoundly affect the porosity of microfibers. The radius of the polymer stream within the sheath flow can be described as a function of the volume flow rates of the polymer and sheath streams. The coaxial flow of appropriate parameters (for example, viscosity, flow rate and channel dimension) produces a laminar flow profile. ${ }^{5}$ The linear velocity, $V(r)$, for laminar flow in a circular outlet tube is given as a function of the distance to the stream center, $r$, where $R$ is the tube radius.

$$
V(r)=\frac{2 Q}{\pi R^{2}}\left(1-\frac{r^{2}}{R^{2}}\right)
$$

The total volume flow rate, $Q_{\text {total }}$, is given by the sum of the polymer volume flow rate, $Q_{\text {polymer }}$ and sheath volume flow rate, $Q_{\text {sheath }}$. As the polymer stream is circular and centered in the tube, the polymer stream volume flow rate must equal the integral of Equation (1) over the area of the polymer stream. Performing the integration and solution for the polymer radius, $R_{\mathrm{s}}$ yields the following equation for the radius of the polymer flow as a function of the polymer stream and total volume flow rates:

$$
R_{\mathrm{s}}=R\left[1-\left(\frac{Q_{\text {sheath }}}{Q_{\text {polymer }}+Q_{\text {sheath }}}\right)^{1 / 2}\right]^{1 / 2}
$$

In the case of our phase-separating multicomponent polymer (triblock copolymer) with various dimensions for confined microfluidic geometry, the preferential wetting of surfaces by one component strongly influences phase-separation behavior. This wetting phenomenon induces 'surface-directed spinodal decomposition', which results in porous structures. We hypothesize that porosity could result from wall effects related to the interplay between wall-surface wetting and polymer-particle interfacial tension and the relative dynamics of diffusion-based transport. In contrast, experimental results indicate that microfibers of DMSO show less porosity than those of $\mathrm{CH}_{2} \mathrm{Cl}_{2}$, which could be due to the higher evaporation rate under spatial restriction for $\mathrm{CH}_{2} \mathrm{Cl}_{2}$. Where liquidliquid demixing occurs, the polymer solution yields polymer-rich and polymer-lean phases. Further evaporation of the solvent leads to an increase in the concentration and vitrification of the polymer, which results in porosity. ${ }^{6}$ The lower porosity of fibers obtained using DMSO as the solvent system could be a result of its nonvolatile nature; however, the confined geometry of the outlet pipette had a significant impact on porosity as a result of spatial restriction.

\section{RESULTS AND DISCUSSION}

Figure 2 shows the field electron scanning electron microscopy images of microfibers obtained using $\mathrm{CH}_{2} \mathrm{Cl}_{2}$ (a) and DMSO (b) as solvent systems, which clearly show the porous nature of microfibers. In this paper, we report that the capillary dimension of the 
It is well known that cell adhesion and proliferation not only depend on scaffold design and structure but also relate to other scaffold surface factors; thus, the evaluation of surface roughness and hydrophilicity is significant in our discussions. Here, we used three-dimensional morphology atomic force microscopy images to calculate fiber surface roughness and contact angle measurements to elucidate the degree of hydrophilicity. Data are given in Table 1. The higher degree of surface roughness for fibers obtained with $\mathrm{CH}_{2} \mathrm{Cl}_{2}$ could be due to the higher porous structures, and these characteristics are of great interest for various biomedical applications. The values of contact angle measurements reflect the higher degree of hydrophilicity of the scaffold with respect to the cell medium. From this result, we believe that our scaffold could potentially favor cell adhesion and proliferation. Furthermore, we encapsulated bovine serum albumin and methylene blue in porous fibers to demonstrate their controlled release (Figure 3). Both show a burst release profile within $1 \mathrm{~h}$ and a controlled/sustained release until $48 \mathrm{~h}$. It is anticipated that porous structures can be used as reservoirs for proteins/drugs that promote cell adhesion and proliferation.

The continuous fiber fabrication using the PDMS based microfluidic device is shown in the supporting movie file (Supplementary Movie). The spontaneously extruded polymeric microfibers with wavy motion coming out from outlet micropipette are clearly shown in the video.

\section{ACKNOWLEDGEMENTS}

This work was supported by a grant (Grant no: R33-2008-000-10067-0) from the WCU program of the Korean Ministry of Education and Science Technology, Republic of Korea.

\section{Mohana Marimuthu and Sanghyo Kim \\ College of Bionanotechnology, Kyungwon University,}

San-65, Bokjeong dong, Gyeonggi-do, Republic of Korea E-mail:samkim@kyungwon.ac.kr

1 Rujitanaroj, P., Pimpha, N. \& Supaphol, P. Wound-dressing materials with antibacterial activity from electrospun gelatin fiber mats containing silver nanoparticles. Polymer 49, 4723 (2008).

2 Pornsopone, V., Supaphol, P., Rangkupan, R. \& Tantayanon, S. Electrospun methacrylate-based copolymer/indomethacin fibers and their release characteristics of indomethacin. Polym Res. 14, 53 (2007).

3 Bahadur, R. K. C., Bhattarai, S. R., Aryal, S., Khil, M. S., Dharmaraj, N. \& Kim, H. Y. Novel amphiphilic triblock copolymer based on PPDO, PCL, and PEG:Synthesis, characterization, and aqueous dispersion. Colloids Surf. A. 292, 69 (2007).

4 Hwang, C. M., Khademhosseini, A., Park, Y., Sun, K. \& Lee, S. H. Microfluidic chip-based fabrication of PLGA microfiber scaffolds for tissue engineering. Langmuir 24, 6845 (2008).

5 Zarrin, F. \& Dovichi, N. J. Sub-picoliter detection with the sheath flow cuvette. Anal. Chem. 57, 2690 (1985).

6 Qi, Z., Yu, H., Chen, Y. \& Zhu, M. Highly porous fibers prepared by electrospinning a ternary system of nonsolvent/solvent/poly(I-lactic acid). Mater. Lett. 63, 415 (2009).

Supplementary Information accompanies the paper on Polymer Journal website (http://www.nature.com/pj) 